\title{
PDRs and XDRs: Theory and Observations
}

\author{
Amiel Sternberg \\ School of Physics \& Astronomy, Tel Aviv University, Ramat Aviv, 69978, ISRAEL \\ amiel@wise.tau.ac.il
}

\begin{abstract}
I present an overview of theory and observations of FUV-photon and X-ray dominated regions (PDRs and XDRs), with a discussion of some recent results.
\end{abstract}

Keywords. PDRs - XDRs

\section{Introduction}

In this talk I present an overview of interstellar "photon-dominated regions" or "photodissociation regions" (PDRs), and "X-ray dominated regions" (XDRs), with a discussion of both theory and observations. The reader is invited to read this document while clicking through the power-point presentation. $\dagger$

PDRs are most broadly defined as neutral components of the interstellar medium (ISM) where far-ultraviolet (FUV; 6-13.6 eV) radiation from external sources, usually massive hot $\mathrm{OB}$ stars and clusters, dominates the gas heating, and controls the chemical structure. In PDRs, the FUV photon penetration depth is limited by dust absorption and scattering, and the gas is heated primarily by photoelectric emission of electrons from grain surfaces. The chemistry is driven by FUV ionization of species with ionization thresholds longward of the hydrogen Lyman limit. In contrast (slide 2) XDRs refer to neutral hydrogen clouds in which keV X-rays dominate the gas heating and chemistry. In XDRs the X-ray penetration is limited by photoionization of the heavy elements ("metals") rather than by dust particles. For this reason the photon penetration lengths in XDRs are much larger than in PDRs. In XDRs the gas is heated by direct photoionization of $\mathrm{H}$ and $\mathrm{H}_{2}$. As I will explain, this leads to more efficient gas heating in XDRs compared to PDRs.

PDRs include the classical "warm neutral medium" (WNM) and "cold neutral medium" (CNM), optically thin diffuse clouds, thicker translucent clouds with moderate visual extinctions $\left(A_{V} \lesssim 5\right)$ and intermediate gas densities $\left(n_{\mathrm{H}} \lesssim 10^{3} \mathrm{~cm}^{-3}\right)$, and optically thick interfaces in dense $\left(n_{\mathrm{H}} \gtrsim 10^{3} \mathrm{~cm}^{-3}\right)$ molecular clouds with high $\left(A_{V} \sim 10\right)$ visual extinctions (slide 3 ). Dense PDRs exposed to intense FUV fields in star-forming regions are of particular interest as they are sources of luminous millimeter, submillimeter, and far-infrared line emissions, such as O I $63 \mu \mathrm{m}$ and C II $157.7 \mu \mathrm{m}$ fine-structure lines, and rotational and vibrational lines of a variety of molecules including the most abundant $\mathrm{CO}$ and $\mathrm{H}_{2}$ molecules. XDRs may be prominent near intense X-ray sources such as accreting black holes in active galactic nuclei.

The study of PDRs, and subsequently XDRs, dates back to the late 70s and early 80s when advances in detector technology opened up the submillimeter and far-infrared spectral windows. In this talk I will comment on some recent results and puzzles. Comprehensive reviews have been presented by Hollenbach \& Tielens (1997, 1999). 


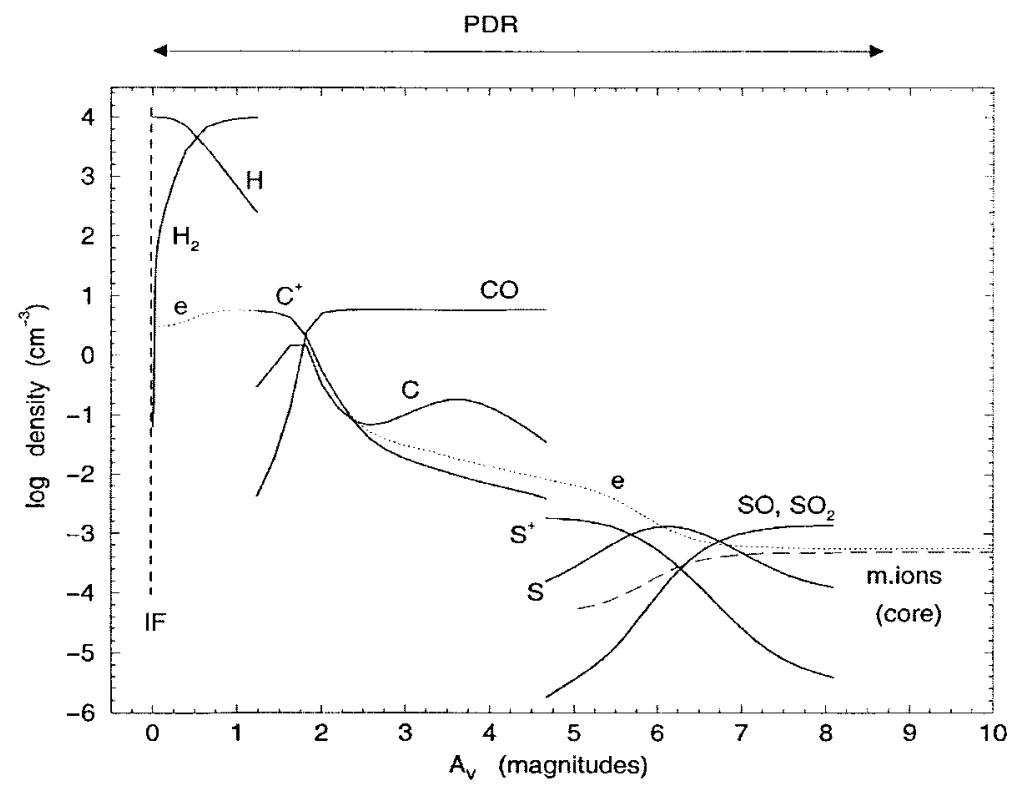

Figure 1. Schematic structure of a PDR. FUV radiation is incident from the left.

\section{Physical Processes}

The starburst galaxy M82 (3.3 Mpc) provides a nice example of a global PDR (slide 4). Optical imaging shows a central dusty starburst with large-scale $H \alpha$ filaments extending out of the galaxy in a galactic superwind driven by the starburst. Infrared, submillimeter, and millimeter-wave spectroscopy of this source reveals a thermal dust continuum, and a wealth of PDR emission lines. Of particular importance are the observed fine-structure line strengths, with line-to-continuum ratios of $0.1-1 \%$. This is typical of many Galactic and extragalactic PDRs.

The PDR line strengths may be understood by considering theoretical models. In the simplest picture (Fig. 1; slide 5) a dense PDR may be considered as a plane-parallel slab of gas that absorbs FUV radiation incident from one side. Any UV radiation capable of ionizing hydrogen is assumed to be absorbed in a thin ionization front adjacent to the PDR. The penetrating FUV radiation dissociates molecules, and ionizes atoms, as it penetrates the cloud. The photorates are attenuated with increasing cloud depth, or visual extinction, as the FUV radiation is absorbed by the dust. The energy absorbed by the dust is reradiated as thermal infrared. Hence atoms such as oxygen (IP $13.62 \mathrm{eV}$ ) remain neutral in the PDR, but atoms such as carbon (IP $11.26 \mathrm{eV}$ ) are singly ionized (in the outer parts). PDRs consist of several distinct zones and transition layers, from outer photodissociated gas to inner fully molecular gas (Jansen et al. 1995; Sternberg \& Dalgarno 1995). The $\mathrm{H} / \mathrm{H}_{2}$ transition occurs closest to the cloud surface where the combined effects of dust opacity and $\mathrm{H}_{2}$ absorption-line "self-shielding" reduce the photodissociation rate. The $\mathrm{H} / \mathrm{H}_{2}$ transition point depends on the efficiency with which $\mathrm{H}_{2}$ forms on the grain surfaces. The transition from $\mathrm{C}^{+}$to $\mathrm{C}$ to $\mathrm{CO}$ occurs deeper in the cloud, and a similar $\mathrm{S}^{+}$to $\mathrm{S}$ to $\mathrm{SO}, \mathrm{SO}_{2}, \mathrm{CS}$ transition zone occurs at still larger $A_{V}$. The sizes of the various transition layers are controlled by several parameters, including most importantly the cloud density (or pressure), and the intensity of the illuminating radiation field. Additional controlling parameters are the grain FUV scattering properties, the cloud geometry and clumpiness, and the elemental gas-phase abundances. 
The FUV intensity is a crucial paramter. Near hot OB stars in star-forming regions the FUV fields can be orders of magnitude more intense than the mean value in the ISM. The FUV intensity is usually given in terms of a scaling factor $\left(G_{0}\right)$ relative to the empirically based mean value in the ISM in the solar neighborhood (Habing 1968; Draine 1978; slide 6). Recent $a b$ initio computations of the interstellar FUV field (Parravano, Hollenbach \& McKee 2003) show that dust opacity limits the contributing OB associations out to distances of $500 \mathrm{pc}$ from typical points within the Galactic disk. The mean FUV field fluctuates in response to the birth and death of OB associations. Intense fields near individual clusters can also vary on shorter timescales (Myr) as the massive stars evolve (Sternberg, Hoffmann \& Pauldrach 2003; slide 7).

The primary gas heating mechanim in PDRs is photoelectric emission from dust grains, first considered by Spitzer (1948) and investigated in many subsequent studies (Draine 1978; Bakes \& Tielens 1994; Weingartner \& Draine 2001). In this process energetic electrons are ejected from the grains at a rate that depends on the FUV intensity and the grain charge, set by the balance between photoemission and recombination (slide 8). A second important process, occurring in dense $\left(n \gtrsim 5 \times 10^{4} \mathrm{~cm}^{-3}\right)$ gas, is the collisional deexcitation of FUV pumped $\mathrm{H}_{2}$ (Sternberg \& Dalgarno 1989).

The gas is cooled by neutral impact excitations of atomic fine-structure, and molecular rotational energy levels, followed by radiative decays. The gas temperature is then set by the balance between heating and cooling. Predicted, and observed, gas temperatures range from a few $100 \mathrm{~K}$ up to $1000 \mathrm{~K}$ in the outer cloud layers, down to several 10 $\mathrm{K}$ at large depths where the FUV is fully attenuated and where other process such as cosmic-ray heating dominates.

Fine-structure cooling plays a particularly important role in PDRs. Indeed the first PDR models (Tielens \& Hollenbach 1985) were constructed in order to account for the observed strengths of fine-structure emission lines in PDRs. A famous example is the ${ }^{2} P_{1 / 2}{ }^{2} P_{1 / 2} 157.7 \mu \mathrm{m}$ line of $\mathrm{C}^{+}$. Long before it was first detected in Orion (Russell et al. 1980), the C II line was recognized theoretically as a likely major cooling transition for the neutral hydrogen component of the ISM (Spitzer 1948; Dalgarno and McCray 1972). $\mathrm{C}$ II cooling plays a crucial role in the theory of the two-phase CNM/WNM structure of the H I gas in the ISM (Field, Goldsmith, \& Habing 1969; Wolfire, McKee, \& Hollenbach 2003), dominating the energy losses of the cool phase. However, the early theoretical considerations focussed primarily on the relatively low density gas $\left(n_{\mathrm{H}} \lesssim 50 \mathrm{~cm}^{-3}\right)$, where the excited level is subthermally populated and the $\mathrm{C}$ II emissivities are low (Fig. 2; slide 9). For fixed density, the cooling efficiency decreases sharply for temperatures $T$ below the transition energy of $92 \mathrm{~K}$, and levels off at higher $T$, where the Boltzmann factor in the collisional excitation rate coefficient approaches unity. At fixed gas temperature the efficiency increases linearly with density up to the critical hydrogen density of $10^{4} \mathrm{~cm}^{-3}$, above which collisional deexcitations quench the radiative emissions. For conditions in the classical WNM $\left(n_{\mathrm{H}} \sim 0.4 \mathrm{~cm}^{-3}, T \approx 8000 \mathrm{~K}\right)$ and $\mathrm{CNM}\left(n_{\mathrm{H}} \approx 60 \mathrm{~cm}^{-3}, T \approx 50 \mathrm{~K}\right)$, the $\mathrm{C}$ II emission efficiencies are well below the maximum possible. However, for densities greater than $10^{2}$ and up to at least $10^{4} \mathrm{~cm}^{-3}$, and for $T$ above $\sim 10^{2} \mathrm{~K}$, the cooling efficiencies are significantly enhanced. This range of elevated density and temperature corresponds to conditions characteristic of dense PDRs.

Thus, in PDRs, most of the incident FUV energy is absorbed by dust particles and reradiated as quasi-thermal IR, but a small fraction of the FUV heats the gas via the photoelectric ejection of electrons from the grain surfaces. The typical "work-function", for interstellar grains is $W=6 \mathrm{eV}$, and the expected photoelectic yield, or the fraction of electrons that are actually dislodged off the grains, is typically $Y=0.1$. Therefore, for a mean incident FUV photon energy of $10 \mathrm{eV}$, a fraction $([10-6] / 10) \times 0.1=0.04$ of 


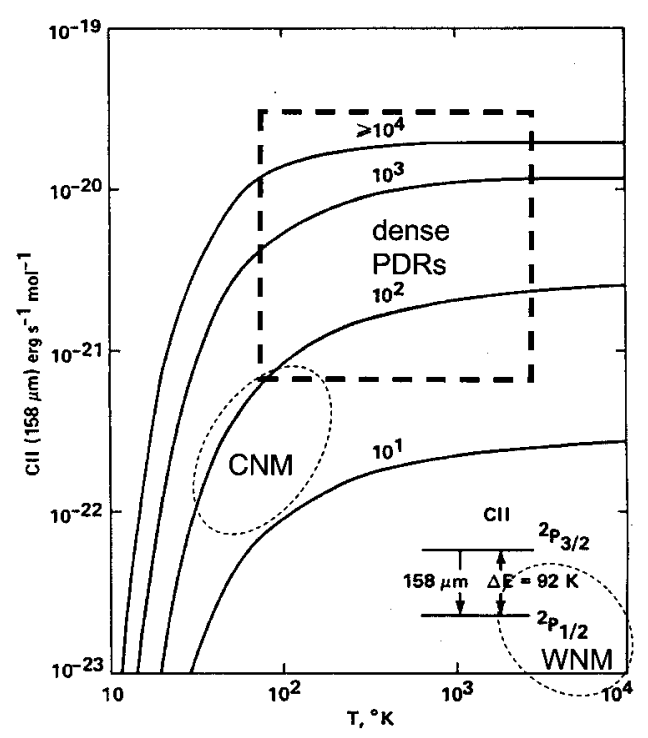

Figure 2. C in line cooling per particle as a function of the gas temperature and hydrogen density, for neutral impact excitation (adapted from Tielens \& Hollenbach 1985).

the original photon energy is available to heat the gas (slide 10). This fraction, or "heating efficiency", decreases if the grains become positively charged, as they do when the photoelectron emission rates become large relative to the grain-electron recombination rates. This argument predicts cooling emission lines with intensities of order $1 \%$ of the thermal IR dust continua, consistent with observations. Photoelectric heating is probably dominated by the smallest $(<15 \AA)$ grains or PAHs for typical grain size distributions (Bakes \& Tielens 1994; Weingartner \& Draine 2001).

The fine-structure line intensties are, therefore, probes of the incident FUV fluxes. For fixed density the line intensities saturate with increasing $G_{0}$ as the heating efficieny decreases (Tielens \& Hollenbach 1985; Sternberg \& Dalgarno 1989). Line ratios, such as [O I] $63.2 \mu \mathrm{m} /[\mathrm{C}$ II $] 157.7 \mu \mathrm{m}$, are sensitive to the density, because $\mathrm{O}$ I is quenched at higher density than is C II (e.g., Kaufmann et al. 1999; slide 11).

\section{PDR Chemistry, CN/HCN}

The $\mathrm{C}^{+} / \mathrm{C} / \mathrm{CO}$ transition layer is one of several locations in PDRs where specific types of molecules are formed (Sternberg \& Dalgarno 1995). The depth at which the $\mathrm{C}^{+} / \mathrm{C} / \mathrm{CO}$ transition occurs depends on the $\mathrm{C}^{+}$recombination efficiency. Because radiative recombination is slow, processes such as mutual neutralization of $\mathrm{C}^{+}$ions with negative $\mathrm{PAH}^{-}$ ions (Lepp \& Dalgarno 1988; Bakes \& Tielens 1998), and "grain assisted" recombination (Weingartner \& Draine 2001) are important (slides 13 and 14).

High abundances of molecular radicals are expected in the outer parts of PDRs where the $\mathrm{C}^{+} / \mathrm{C} / \mathrm{CO}$ transition occurs. One nice example of this is the behavior of $\mathrm{CN} / \mathrm{HCN}$ in PDRs (Boger \& Sternberg 2005). Fuente et al. (1993, 2003) presented molecular maps of the edge on PDR in the reflection nebula NGC 7023. In this object, the illuminating star is a Herbig B3Ve star, and $G_{0}=2.4 \times 10^{3}$ at the PDR interface. The cloud density is about $10^{4} \mathrm{~cm}^{-3}$. Fuente et al. found that the $\mathrm{CN}$ radical peaks closer to the illuminating 


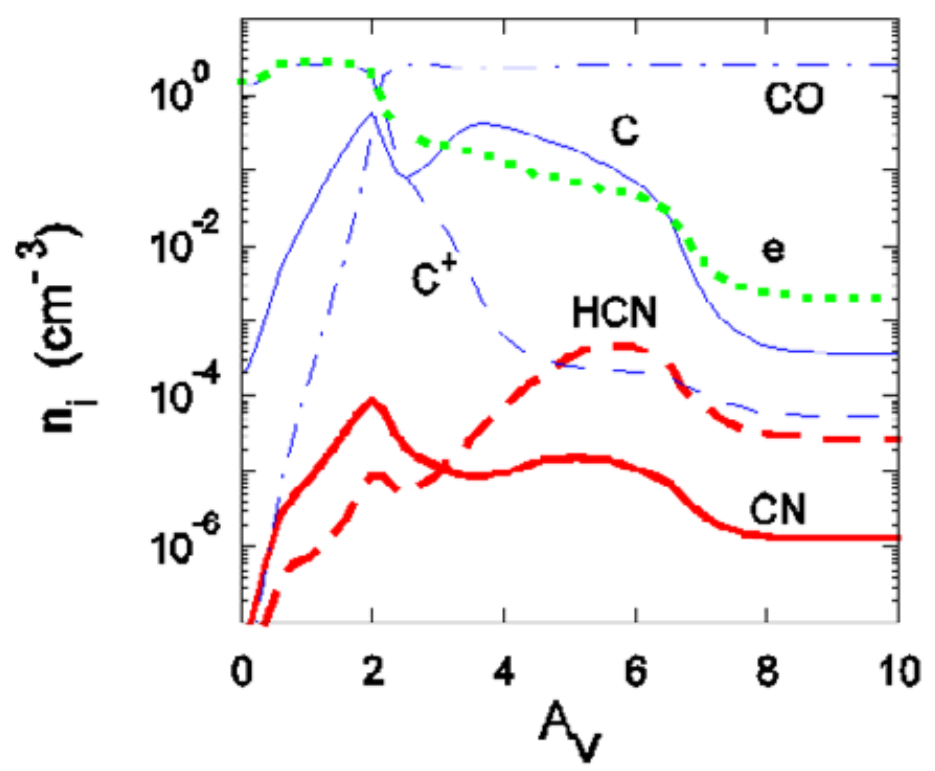

Figure 3. CN and HCN densities as functions of cloud depth, for $G_{0}=10^{3}$ and $n_{\mathrm{H}}=10^{4} \mathrm{~cm}^{-3}$ (adapted from Boger \& Sternberg 2005).

star than does the saturated molecule $\mathrm{HCN}$, and that the $\mathrm{CN} / \mathrm{HCN}$ abundance ratio decreases with increasing cloud depth (slide 15).

The explanation for the CN peak is as follows (Boger \& Sternberg 2005; slides 17 and 18). Because the hydrogen becomes fully molecular within the $\mathrm{C}^{+}$layer, $\mathrm{CN}$ is rapidly produced by a gas-phase sequence initiated by radiative association

$$
\mathrm{C}^{+}+\mathrm{H}_{2} \rightarrow \mathrm{CH}_{2}^{+}+\nu \text {. }
$$

This is then followed by rapid abstraction, and dissociative recombination

$$
\begin{gathered}
\mathrm{CH}_{2}^{+}+\mathrm{H}_{2} \rightarrow \mathrm{CH}_{3}^{+}+\mathrm{H} \\
\mathrm{CH}_{3}^{+}+\mathrm{e} \rightarrow \mathrm{CH}+\mathrm{H}_{2} \\
\mathrm{CH}_{3}^{+}+\mathrm{e} \rightarrow \mathrm{CH}_{2}+\mathrm{H},
\end{gathered}
$$

leading to the radicals $\mathrm{CH}$ and $\mathrm{CH}_{2}$. These then react with abundant $\mathrm{N}$ atoms

$$
\begin{gathered}
\mathrm{CH}+\mathrm{N} \rightarrow \mathrm{CN}+\mathrm{H} \\
\mathrm{CH}_{2}+\mathrm{N} \rightarrow \mathrm{HCN}+\mathrm{H} .
\end{gathered}
$$

The $\mathrm{CN}$ and $\mathrm{HCN}$ molecules are removed by photodissociation

$$
\begin{gathered}
\mathrm{CN}+\nu \rightarrow \mathrm{C}+\mathrm{N} \\
\mathrm{HCN}+\nu \rightarrow \mathrm{CN}+\mathrm{H} .
\end{gathered}
$$

A CN density peak occurs at cloud depths where the $\mathrm{C}^{+}$density is still large, but where the $\mathrm{CN}$ photodissociation rate is attenuated as much as possible, i.e., at the inner edge of the $\mathrm{C}^{+}$zone. Photodissociation keeps the $\mathrm{CN} / \mathrm{HCN}$ ratio high at this location. Because $\mathrm{HCN}$ is more vulnerable to photodissociation than is $\mathrm{CN}$, as the FUV radiation is attenuated, the HCN density grows depth while the $\mathrm{CN}$ density saturates. The $\mathrm{CN} / \mathrm{HCN}$ 


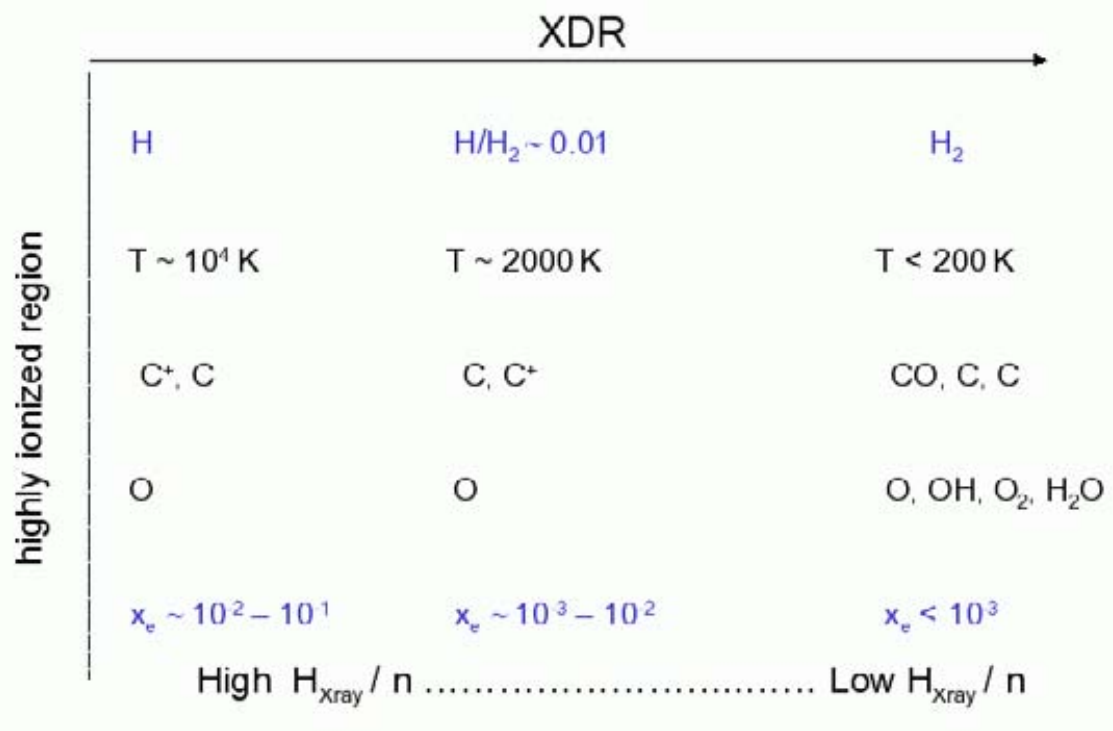

Figure 4. XDR structure (adapted from Maloney et al. 1996).

ratio therefore decreases with depth, and this abundance ratio is a probe of the local FUV field strength. This behavior is displayed in Figure 3.

Additional chemical diagnostics of PDRs are discussed in Sternberg \& Dalgarno (1995).

\section{XDRs}

In X-ray dominated regions (XDRs), penetrating ( $\mathrm{keV}$ ) X-rays heat the gas and drive the chemistry. As already mentioned, there are two important differences compared to PDRs. First, because the X-rays are absorbed in photoionization events, as opposed to the FUV dust absorption that occurs in PDRs, the X-ray intensity falls off much more slowly in XDRs. Second, the radiative heating efficiency, that is, the fraction of X-ray energy converted to heat and then rereadiated in cooling emission lines, is larger in XDRs than in PDRs.

As discussed by Maloney et al. (1996) a key parameter in XDRs is the ratio, $H_{\mathrm{X}} / n_{\mathrm{H}}$, of the local X-ray energy deposition rate $H_{\mathrm{X}}$ to hydrogen gas density $n_{\mathrm{H}}$ (slide 19 ). For large $H_{\mathrm{X}} / n_{\mathrm{H}}$, the gas remains primarily atomic, with large $(0.1-0.01)$ ionization fractions, and at high $\left(\sim 10^{4} \mathrm{~K}\right)$ temperature. The $\mathrm{H} / \mathrm{H}_{2}$ ratio, ionization fraction, and gas temperature, decrease as $H_{\mathrm{X}} / n_{\mathrm{H}}$ becomes small. Molecules form with high efficiency at intermediate values of $H_{\mathrm{X}} / n_{\mathrm{H}}$ where the gas temperature is maintained at a level of a few hundred $\mathrm{K}$.

It is of interest to consider the X-ray energy deposition in the limit of a fully molecular gas (slide 20). In this case, energetic electrons are produced by photoionization

$$
\mathrm{H}_{2}+\mathrm{Xray} \rightarrow \mathrm{H}_{2}^{+}+\mathrm{e}^{*} .
$$

The energetic electrons, $e^{*}$, lose energy via Coulomb scattering with free thermal electrons, by electronic excitations of the $\mathrm{H}_{2}$ molecules (and trace $\mathrm{H}$ atoms), and by collisional ionizations of the $\mathrm{H}_{2}$. For small fractional ionizations Couloumb scattering is a minor channel. The excitations are followed by emissions of UV photons which are 
absorbed by, and heat, the dust. The induced UV photons also mediate the chemistry by photodissociating molecules. Importantly, the electron impact ionizations

$$
\mathrm{e}^{*}+\mathrm{H}_{2} \rightarrow \mathrm{H}_{2}^{+}+\mathrm{e}+\mathrm{e}^{*}
$$

are immediatley followed by proton transfer

$$
\mathrm{H}_{2}^{+}+\mathrm{H}_{2} \rightarrow \mathrm{H}_{3}^{+}+\mathrm{H}
$$

and dissociative recombination

$$
\mathrm{H}_{3}^{+}+\mathrm{e} \rightarrow \mathrm{H}_{2}+\mathrm{H}
$$

Most of the ionization energy of $15.4 \mathrm{eV}$ is converted into kinetic energy of the dissociating fragments, i.e., heats the gas (Glassgold \& Langer 1973). However, the energy lost to each energetic electron per ionization event is on average $37.7 \mathrm{eV}$ (Dalgarno et al. 1999). Therefore a heating efficiency of about $15.4 / 37.7=0.4$ is expected in XDRs, significantly larger than in PDRs. This effect is seen in computations of the fine-structure emission line intensities in XDRs, with large resulting line-to-continuum ratios (slide 21).

Dale et al. (2004) suggest that the enhanced [O I] $63 \mu \mathrm{m}$ emission they observed in a sample of Seyfert galaxies arises in XDRs.

\section{Galaxies}

\subsection{PDRs}

Another nice example of a PDR is the starburst in the "Antennae" pair of colliding galaxies NGC 4038/4039 (slides 22 and 23). Optical HST imaging (Whitmore \& Schweizer 1995) shows that the system contains hundreds of compact clusters of young OB stars that are interacting with surrounding dense and dusty molecular clouds. Near-IR Keck spectroscopy of the embedded mid-IR star cluster detected in ISO observations reveals a spectrum containing a wealth of vibrational $\mathrm{H}_{2}$ emission lines (Gilbert et al. 2000; slide 23). The luminosity in just the $1-0 \mathrm{~S}(1)$ line alone is $9600 L_{\odot}$. The relative intensities of lines in the $v=1$ and $v=2$ bands indicate that the $\mathrm{H}_{2}$ excitation mechanism is FUVpumping (slide 24). In this process FUV photons are absorbed in the Lyman and Werner bands, followed by rapid radiative decays to excited vibrational levels of the ground electronic state. These then decay in a cascade of quadrupole transitions giving rise to a rich infrared spectrum (Black \& Dalgarno 1976; Black \& van Dishoeck 1987; Sternberg 1988; Sternberg \& Dalgarno 1989; Draine \& Bertoldi 1996; Shaw et al. 2005). The orthoto-para ratio in excited states is $1.6 \pm 0.07$. This is nicely consistent with what is expected for FUV-pumping in optically thick absorption lines, for a ground vibrational state with an equilibrium rotational ortho-to-para ratio of 3 indicative of warm gas (Sternberg \& Neufeld 1999).

Far-infrared imaging spectroscopy of the Antennae shows that the C II line emission peaks at the location of the "interaction zone" located between the individual galaxy nuclei (Nikola et al. 1998; slide 26). A global FUV intensity of $G_{0} \approx 500$ is inferred for the interaction zone. The FUV radiation is interacting with a total gas mass of at least a few $10^{7} \mathrm{M}_{\odot}$. The total luminosity in the $\mathrm{C}$ II line is $3.7 \times 10^{8} \mathrm{~L}_{\odot}$, corresponding to about $1 \%$ of the far-IR (IRAS) luminosity.

The C II to FIR luminosity ratio in the Antennae pair is consistent with expectations from PDR theory. This contrasts with recent observations of more luminous systems. For example, Maiolino et al. (2005) recently detected the [C II] line in the high redshift, $z=6.42$, quasar J1148+5251 (slide 26). The observations were carried out at the IRAM 

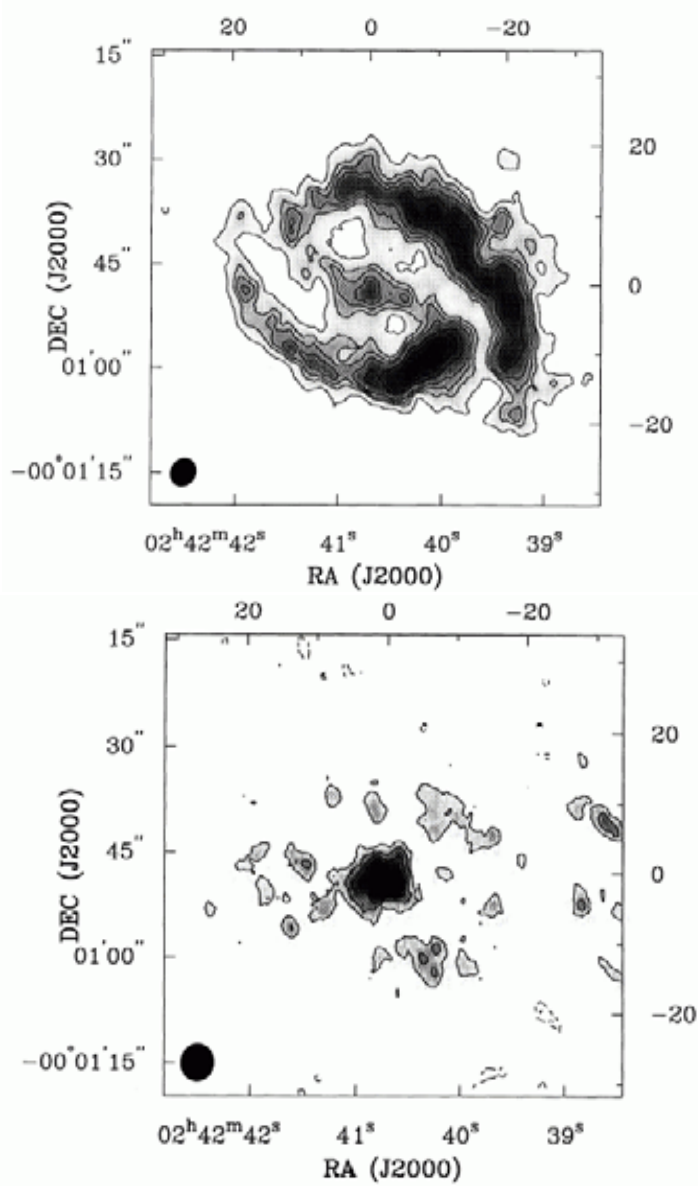

Figure 5. CO (top) and HCN (bottom) in NGC 1068 (from Helfer \& Blitz [1995]).

30-meter telescope. They find $L_{\mathrm{FIR}}=2.2 \times 10^{13} \mathrm{~L}_{\odot}$, and $L_{\mathrm{CII}}=4.4 \times 10^{9} \mathrm{~L}_{\odot}$, and infer a total star-formation rate of $3000 \mathrm{M}_{\odot} \mathrm{yr}^{-1}$. Interestingly, the small FIR to C II ratio of $2 \times 10^{-4}$ is comparable to what is observed in ultraluminous infrared galaxies (Luhman et al. 2003; slide 27). Possible explanations for the C II "line deficit" in these objects include (a) large ratios of FUV flux to gas density in the ULIRG (and quasar) PDRs leading to inefficient photoelectric gas heating, (b) an (unlikely) underabundance of OB stars relative to cooler stars, (c) line "self absorption" in optically thick clouds, and (d) a significant absorption of FUV photons in dusty H II regions with large ionization parameters. This last possibility is favored by Luhman et al. (2003), although it remains unclear whether this idea is compatible with the nebular emission line spectra from photoionized gas in these objects.

\subsection{XDRs}

For some examples of extragalactic XDRs, I first turn to the the active galaxy NGC 4258 (7.3 Mpc; slide 28). This object is famous for the prominent $22 \mathrm{GHz} \mathrm{H}_{2} \mathrm{O}$ water masers that have been resolved via VLBI in a rotating circumnuclear disk (e.g., Herrnstein et al. 2005; slide 29). The Keplerian orbital motions imply that the central supermassive black hole mass is $3 \times 10^{7} \mathrm{M}_{\odot}$. Neufeld et al. (1994) suggested that the water masers are produced in XDRs formed in molecular material exposed to the X-rays emitted by the 


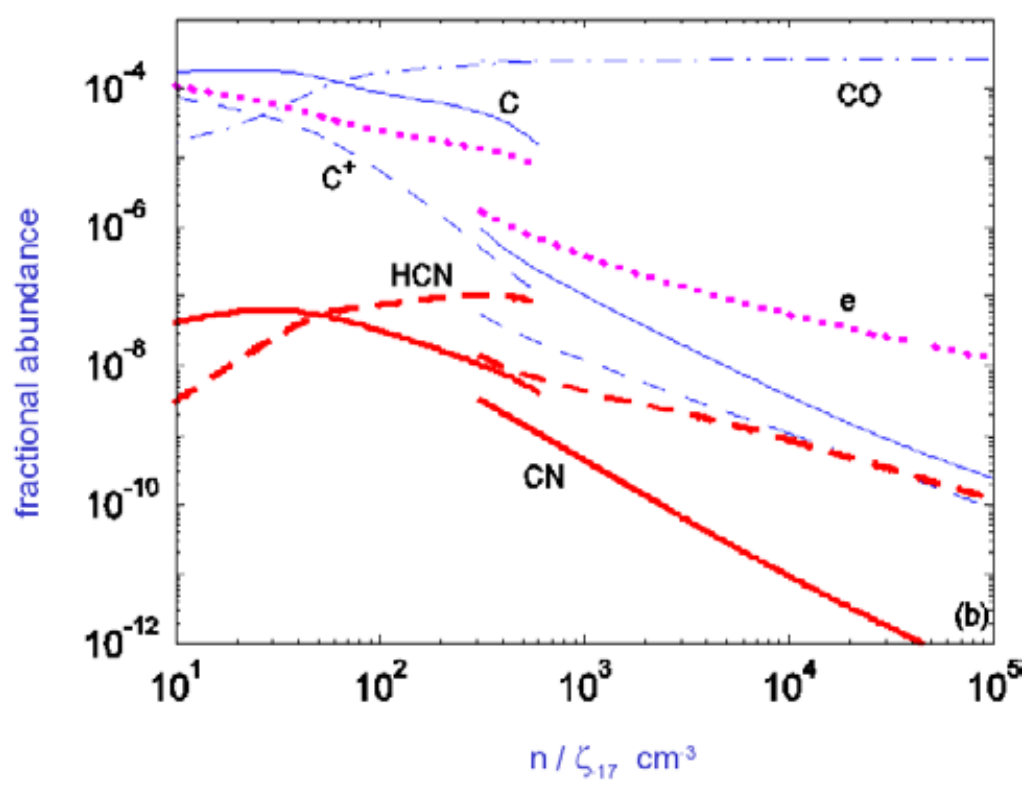

Figure 6. CO (top) and HCN (bottom) in NGC 1068 (from Helfer \& Blitz 1995).

accreting black hole (slide 30). In this model water is formed efficiently via the direct neutral-neutral reactions

$$
\begin{gathered}
\mathrm{O}+\mathrm{H}_{2} \rightarrow \mathrm{OH}+\mathrm{H} \\
\mathrm{OH}+\mathrm{H}_{2} \rightarrow \mathrm{H}_{2} \mathrm{O}+\mathrm{H}
\end{gathered}
$$

in the X-ray heated gas (slide 31). For temperatures above $300 \mathrm{~K}$, the above sequence drives a large fraction of the available oxygen into water. In cooler gas, $\mathrm{H}_{2} \mathrm{O}$ is formed less efficiently via ion-molecule reactions (e.g., Herbst \& Klemperer 1973; Prasad \& Huntress 1980). However, while the kinematic maser disk in NGC 4258 is spectacular, the interpretation in terms of XDRs remains uncertain because the $\mathrm{H}_{2} \mathrm{O}$ abundances cannot be reliably inferred from the nonthermally amplified maser emissions.

The Seyfert 2 galaxy NGC 1068 (15.5 Mpc; slide 32) may be a better source for a quantative study of XDR chemistry. Millimeter-wave interferometry shows that in NGC 1068 molecular emissions are produced in a kpc scale star-forming ring, and in an inner $100 \mathrm{pc}$ scale region surrounding the active nucleus (slide 33). A variety of molecules have been detected in the nuclear region, including $\mathrm{CO}, \mathrm{HCN}, \mathrm{CN}, \mathrm{CS}, \mathrm{HCO}^{+}, \mathrm{HOC}^{+}$, and $\mathrm{SiO}$ (Tacconi et al. 1994; Sternberg et al. 1994; Helfer \& Blitz 1995; Usero et al. 2004). The mm-wave maps show clear differences between the circumnuclear ring, and the nucleus. In particular the $\mathrm{HCN} / \mathrm{CO}$ intensity ratio is small in the ring, and strikingly large in the nucleus (Fig. 5; slide 34). Part of this difference can be attributed to a higher gas density in the nucleus which then favors emission from molecules with large dipole moments such as HCN. However, a radiative transfer analysis (Sternberg, Genzel \& Tacconi 1994) shows that the large $\mathrm{HCN} / \mathrm{CO}$ ratio in the nucleus is due in part to an enhanced $\mathrm{HCN} / \mathrm{CO}$ abundance ratio. Usero et al. (2004) infer an $\mathrm{HCN} / \mathrm{CO}$ abundance ratio of $\sim 10^{-3}$ in the nucleus, an extraordinarily large value compared to Galactic molecular clouds.

Lepp \& Dalgarno (1996) suggested that the large HCN/CO abundance ratio in the nucleus of NGC 1068 might be due to an enhanced ionization rate due to X-rays from the accreting black hole. They also suggested that $\mathrm{CN}$ would persist at high ionization rates. 
Figure 6 (slide 35) shows recent computations by Boger \& Sternberg (2005) that support these ideas. Figure 6 shows the computed abundances (relative to $\mathrm{H}_{2}$ ) for several species including $\mathrm{CN}, \mathrm{HCN}$, and $\mathrm{CO}$, as functions of the parameter $n_{H} / \zeta_{-17}\left(\mathrm{~cm}^{-3}\right)$, where $\zeta_{-17}$ is the $\mathrm{H}_{2}$ ionization rate in units of $10^{-17} \mathrm{~s}^{-1}$. The abundances are for a pure gas-phase chemistry in steady state, for Galactic ISM abundances of the heavy elements carbon, nitrogen, and oxygen. At high density or low ionization rate, the gas is in a lowionization phase (LIP). In the LIP the $\mathrm{CN}$ and $\mathrm{HCN}$ abundances, and the $\mathrm{HCN} / \mathrm{CO}$ and $\mathrm{CN} / \mathrm{HCN}$ density ratios, are small. At low density or high ionization rate the gas is in a high-ionization phase (HIP). In the HIP, the CN and HCN abundances become large. Furthermore, HCN/CO approaches $\sim 10^{-3}$ in the HIP, similar to what is observed in the nucleus of NGC 1068. Furthermore, the $\mathrm{CN} / \mathrm{HCN}$ ratio increases with the ionization rate. This is nicely consistent with the recent observations of Usero et al. (2004) who find $\mathrm{CN} / \mathrm{HCN} \gtrsim 1$ in the nucleus of 1068 .

We have seen that large $\mathrm{CN} / \mathrm{HCN}$ ratios are expected in both PDRs and in XDRs, though for somewhat different reasons. This raises the general question of whether there are any unique chemical diagnostics of XDRs that may be used to distinguish them from PDRs, beyond the enhanced ionization rates or large line-to-continuum ratios expected in XDRs. One idea is to make use of the fact that in XDRs multiply charge atomic ions are expected to coexist together with the neutral molecular gas. The multiply charged species are produced by inner-shell X-ray ionization and Auger decay, processes that do not occur in FUV irradiated gas in PDRs. Ions such as $\mathrm{C}^{2+}$ (Dalgarno 1976; Langer 1978) and $\mathrm{S}^{2+}$ (Yan \& Dalgarno 1996) have been put forth as possible candidates, provided they are not rapidly neutralized by charge transfer or gas-grain reactions. Fine structure line transtions of $\mathrm{S}^{2+}$ at 33.5 and $18.7 \mu \mathrm{m}$ would then be potentially useful probes. If these ions react rapidly with $\mathrm{H}_{2}$ they may lead to enhanced $\mathrm{CH}^{+}$and $\mathrm{SH}^{+}$in $\mathrm{XDRs}$ (see also Sternberg, Yan \& Dalgarno 1996).

\section{Conclusion}

The study of PDRs and XDRs will remain important for many years to come, especially with the recent advent of the Spitzer Space Telescope, and other upcoming facilities including SOFIA, ALMA, and Herschel (slide 37).

\section{Acknowledgements}

I thank the organizers for an excellent meeting, and for inviting me to speak. My research in molecular astrophysics is supported by the Israel Science Foundation.

\section{References}

Bakes, E.L.O. \& Tielens, A.G.G.M. 1994, Ap. J. 427, 822

Bakes, E.L.O. \& Tielens, A.G.G.M. 1998, Ap. J. 499, 258

Black, J.H. \& Dalgarno, A. 1976, Ap. J. 203, 132

Black, J.H. \& van Dishoeck, E.F. 1987, Ap. J. 322, 412

Boger, G.I. \& Sternberg, A. 2005, Ap. J. 632, 302

Crawford, M.K., Genzel, R., Townes, C.H., \& Watson, D.M. 1985, Ap. J. 291, 755

Dale, D.A., Helou, G., Brauher, J.R., Cutri, R.M., Malhotra, S., \& Beichman, C.A. 2004, Ap. J. 604,565

Dalgarno, A. \& McCray, R.A. 1972, ARAA 10, 375

Dalgarno, A., Yan, M., \& Weihong, L. 1999, Ap. J. Suppl. 125, 237

Draine, B.T. 1978, Ap. J. Suppl. 36, 395

Draine, B. \& Bertoldi, F. 1996, Ap. J. 468, 269 
Field, G.B., Goldsmith, D.W., \& Habing, H.J. 1969, Ap. J. 155, L149

Fuente, A., Martin-Pintado, J., Cernicharo, J., \& Bachiller, R. 1993, 276, 473

Fuente, A., Rodriguez-Franco, A., Garcia-Burillo, S., Martin-Pintado, J., \& Black, J.H. 2003, $A \mathscr{E} A$ 406, 899

Genzel, R., Watson, D.M., Townes, C.H., Dinerstein, H.L., Hollenbach, D., Lesterm D.F., Werner, M., \& Storey, J.W.V. 1984, Ap. J. 276, 551

Gilbert, A.M. et al. 2000, Ap. J. 533, L57

Glassgold, A.E. \& Langer, W.D. 1973, Ap. J. 186, 859

Goldshmidt, O. \& Sternberg, A. 1995, 439, 256

Habing, H.J. 1969, Bull. Astr. Inst. Netherlands, 19, 421

Helfer, T.T. \& Blitz, L. 1995, Ap. J. 450, 90

Herbst, E. \& Klemperer, 1973, Ap. J. 185, 505

Herrnstein, J.R., Moran, J.M., Greenhill, L.J., \& Trotter, A.S. 2005, Ap. J. 629, 719

Hollenbach, D. \& Tielens, A.G.G.M. 1997, ARAA 35, 179

Hollenbach, D. \& Tielens, A.G.G.M. 1999, Rev. Mod. Phys., 71, 173

Jansen, D.J., Spaans, M., Hogerheijde, M.R., \& van Dishoeck, E.F. 1995, A\&A A 303, 541

Kaufman, M.J., Wolfire, M.G., Hollenbach, D.J., \& Luhman, M.L. 1999, Ap. J. 527, 795

Lepp, S. \& Dalgarno, A. 1988, Ap. J. 335, 769

Lepp, S. \& Dalgarno, A. 1996, A\& A 306, L21

Lugten, J.B., Watson, D.M., Crawford, M.K., \& Genzel, R. 1986, Ap. J. 311, L51

Luhman, M.L., Satyapal, S., Fischer, J., Wolfire, M.G., Sturm, E., Dudley, C.C., Lutz, D., \& Genzel, R. 2003, Ap. J. 594, 758

Maloney, P.R., Hollenbach, D.J., \& Tielens, A.G.G.M. 1996, Ap. J. 466, 561

Maiolino, R. et al. 2005, A\& $A$ 440, L51

McKee, C.F. 1989, Ap. J. 345, 782

Melnick, G., Gull, G.E., \& Harwit, M. 1979, Ap. J. 227, L29

Nikola, T., Genzel, R., Herrmann, F., Madden, S.C., Poglitsch, A., Geis, N., Townes, C.H., \& Stacey, G.J. 1998, Ap. J. 504, 749

Neufeld, D.A., Maloney, P.R., \& Conger, S. 1994, Ap. J. 436, L127

Parravano, A., Hollenbach, D.J., \& McKee, C.F. 2003, Ap. J. 584, 79

Prasad, S.S. \& Huntress, W.T. 1980, Ap. J. Suppl. 43, 1

Russell, R.W., Melnick, G., Gull, G.E., \& Harwit, M. 1980, Ap. J. 240, L99

Shaw, G., Ferland, G.J., Abel, N.P., Stancil, P.C., \& van Hoof, P.A.M. 2005, Ap. J. 624, 794

Spitzer, L.Jr., 1948, Ap. J. 107, 6

Sternberg, A. 1988, Ap. J. 332, 400

Sternberg, A. \& Dalgarno, A. 1989, Ap. J. 338, 197

Sternberg, A., Genzel, R., \& Tacconi, L. 1994, Ap. J. 436, L131

Sternberg, A. \& Neufeld, D. 1999, Ap. J. 516, 371

Sternberg, A. \& Dalgarno, A. 1995, Ap. J. Suppl. 99, 565

Sternberg, A., Yan, M., \& Dalgarno, A. 1996 in Molecules in Astrophysics: Probes 85 Processes, ed. E.F. van Dishoeck (Dordrecht: Kluwer), IAU Symp., 178, p. 141

Sternberg, A., Hoffmann, T., \& Pauldrach A.W.A. 2003, Ap. J. 599, 1333

Storey, J.W.V., Watson, D.M,. \& Townes, C.H., 1979, Ap. J. 233, 109

Tacconi, L.J., Genzel, R., Blietz, M., Cameron, M., Harris, A.I., \& Madden, S. 1994, 426, L77

Tielens, A.G.G.M. \& Hollenbach, D. 1985, 291, 722

Usero, A., Garca-Burillo, S., Fuente, A., Martn-Pintado, J., \& Rodrguez-Fernndez, N.J. 2004, $A \& A 419,897$

Weingartner, J.C. \& Draine, B.T. 2001, Ap. J. Suppl. 134, 263

Whitmore, B.C. \& Schweizer, F. 2005, A. J. 109, 960

Wolfire, M.G., McKee, C.F., Hollenbach, D., \& Tielens, A.G.G.M. 2003, 587, 278 


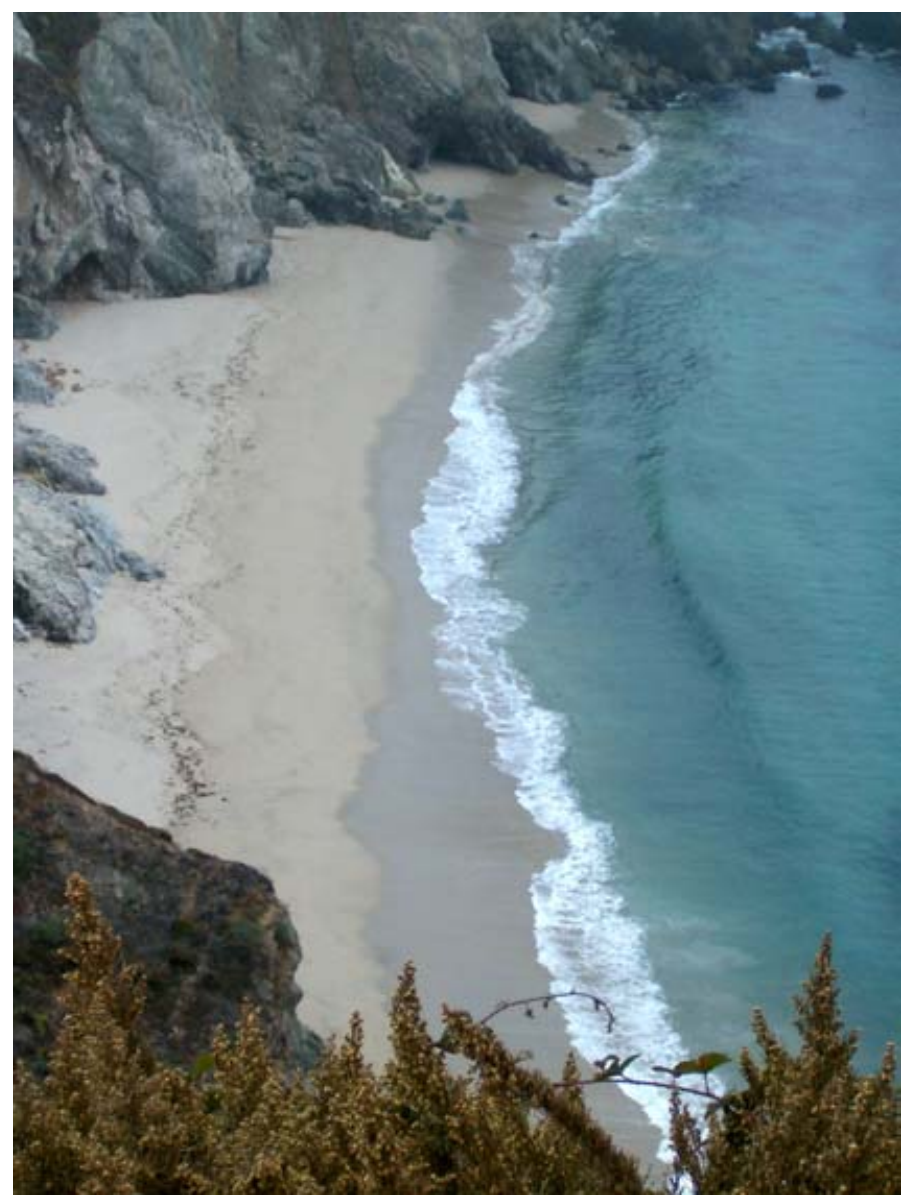

Photo: E. Herbst 